\title{
Pharmacokinetics of Guanfu Base I, an Active Metabolite of Guanfu Base A, in Rats
}

\author{
Xiaotian LI, Guangji Wang, ${ }^{*}$ Sujun Wang, Jianguo Sun, and Jinghan LiU \\ Key Laboratory of Drug Metabolism \& Pharmacokinetics, China Pharmaceutical University; Nanjing 210009, China. \\ Received January 11, 2005; accepted March 22, 2005
}

\begin{abstract}
A liquid chromatographic-mass spectrometric (LC-MS) method was developed and validated for determination of guanfu base I (GFI), and the pharmacokinetics of GFI in Sprague-Dawley rats was examined. The method was linear in the $0.05-20 \mu \mathrm{g} / \mathrm{ml}$ concentration range $(r=0.9994)$. The recovery of guanfu base $I$ was more than $80 \%$. The intraday and interday precision, expressed as the relative standard deviation (RSD), was generally good $(<15 \%)$. After i.v. dosing, plasma GFI concentration declined in a bi-phasic manner with a terminal elimination half-life of $2.49 \mathrm{~h}$. The total plasma clearance values was $1.46 \mathrm{l} / \mathrm{h} / \mathrm{kg}$. After oral dosing, the plasma GFI concentration reached a maximum within $0.5 \mathrm{~h}$. The absolute bioavailability of GFI was $71.31 \%$.
\end{abstract}

Key words guanfu base I; pharmacokinetics; LC-MS

Guangfu base A (GFA, Fig. 1) is a novel anti-arrhythmic drug isolated from the root of Aconitum coreanum (LEVL.) RAPAICS, ${ }^{1)}$ and is currently undergoing phase IV clinical study. GFA has been shown to have potent anti-arrhythmic activity with low toxicity in animal experiments. ${ }^{2,3)}$ Electrophysiological experiments have revealed that GFA blocks the fast $\mathrm{Na}^{+}$channel and exhibits anti-arrhythmic action via a direct effect on the sinoatrial node. ${ }^{4-6)}$ It has been reported that GFA can be metabolized to GFI in rats and humans, ${ }^{7,8)}$ and that GFI also has a potent anti-arrhythmic effect. ${ }^{9}{ }^{9}$ The study of the action of GFA requires a good understanding of the pharmacokinetics of the parent compounds and their metabolites, however, the pharmacokinetics of GFI have not been reported. Therefore, the objective of this study was to develop and validate a LC-ESI-MS method in order to examine the pharmacokinetics of GFI after single intravenous and oral doses to rats.

\section{MATERIALS AND METHODS}

Chemicals and Reagents GFI was supplied by the GFA Study Group, China Pharmaceutical University. Phenoprolamine hydrochloride (DDPH, internal standard) was supplied by Prof. L. L. Sheng (Center of Drug Analysis, China Pharmaceutical University). Acetonitrile was of HPLC grade (Fisher, U.S.A.). All other chemicals were of the highest quality or analytical grade.

Liquid Chromatographic Conditions Mobile phase: $0.2 \%$ acetic acid-acetonitrile $(30: 70 \mathrm{v} / \mathrm{v})$; Column: Shi-

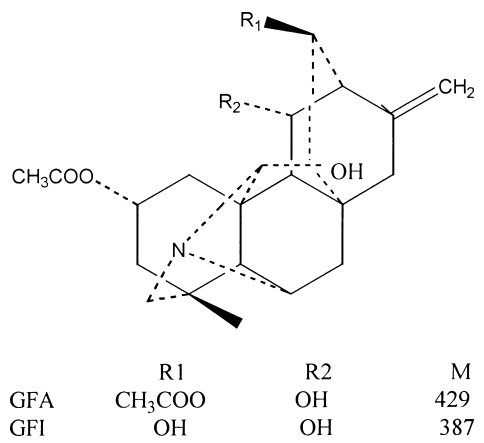

Fig. 1. Structure of Guanfu Base A and Guanfu Base I madzu VP-ODS, C8, $250 \mathrm{~mm} \times 2.1 \mathrm{~mm}$ ID, maintained at $40^{\circ} \mathrm{C}$; flow rate: $0.2 \mathrm{ml} / \mathrm{min}$.

Mass Spectrometry Detection The LC-MS2010 A series system (Shimadzu, Japan) was equipped with a binary pump, on-line vacuum degasser, autosampler, column compartment, diode array detector, mass spectrometry detector of electrospray interface, and LCMS Solution Version 2.04 S. The ESI ion source was set in the positive ion polarity mode for acquiring all mass spectrometry data. The selective ion monitoring (SIM) was set at $m / z 388$ for GFI and $m / z 344$ for DDPH. The drying gas flow, CDL temperature, block heater temperature, $\mathrm{CDL}$ voltage, probe voltage, and detector voltage were set to $1.5 \mathrm{l} / \mathrm{min}, 250^{\circ} \mathrm{C}, 200^{\circ} \mathrm{C}, 25 \mathrm{~V}, 4.5 \mathrm{kV}$, and $1.65 \mathrm{kV}$, respectively.

Rat Samples Male Sprague-Dawley rats $(180-220 \mathrm{~g})$ were purchased from Shanghai Experimental Animal Center, Chinese Academy of Science, and used in the study after a 1week acclimatization period. After overnight fasting, guanfu base I hydrochloride was administered intravenously (i.v.) or orally at doses of $20 \mathrm{mg} / \mathrm{kg}$ to each group of rats for the plasma concentration-time course study. Blood samples (about $0.25 \mathrm{ml}$ ) were collected in heparinized tubes pre-dose $(0 \mathrm{~h})$ and at $0.08,0.17,0.25,0.5,1,2,4,6,8,10$, and $12 \mathrm{~h}$ after dosing. Plasma was immediately separated by centrifugation at $3000 \times \boldsymbol{g}$ and stored in polypropylene tubes at $-20^{\circ} \mathrm{C}$ until analysis.

Sample Extraction Plasma $(0.1 \mathrm{ml})$ the internal standard solution $20 \mu \mathrm{l}(5 \mu \mathrm{g} / \mathrm{ml})$ and $40 \mu \mathrm{l}$ of saturated $\mathrm{Na}_{2} \mathrm{CO}_{3}$ were added to a test tube. The sample was mixed and then extracted with $1 \mathrm{ml}$ of ethyl acetate using a vortex for $3 \mathrm{~min}$, and centrifuged at $3000 \times \boldsymbol{g}$ for $5 \mathrm{~min}$. The top organic layer $(0.5 \mathrm{ml})$ was transferred to another tube and evaporated to dryness at $50{ }^{\circ} \mathrm{C}$ under a gentle stream of nitrogen. The residue was reconstituted in $0.1 \mathrm{ml}$ of mobile phase, and centrifuged at $10000 \times \boldsymbol{g}$ at $4{ }^{\circ} \mathrm{C}$ for $10 \mathrm{~min}$. The supernatant (101) was injected into the LC-MS.

Data Analysis The peak areas of guanfu base I and the internal standard were measured. The peak area ratios of guanfu base I relative to that of the internal standard were calculated and used for construction of the standard curves. These peak area ratios were plotted against the added concentration of each of the standards. Least-square linear re- 

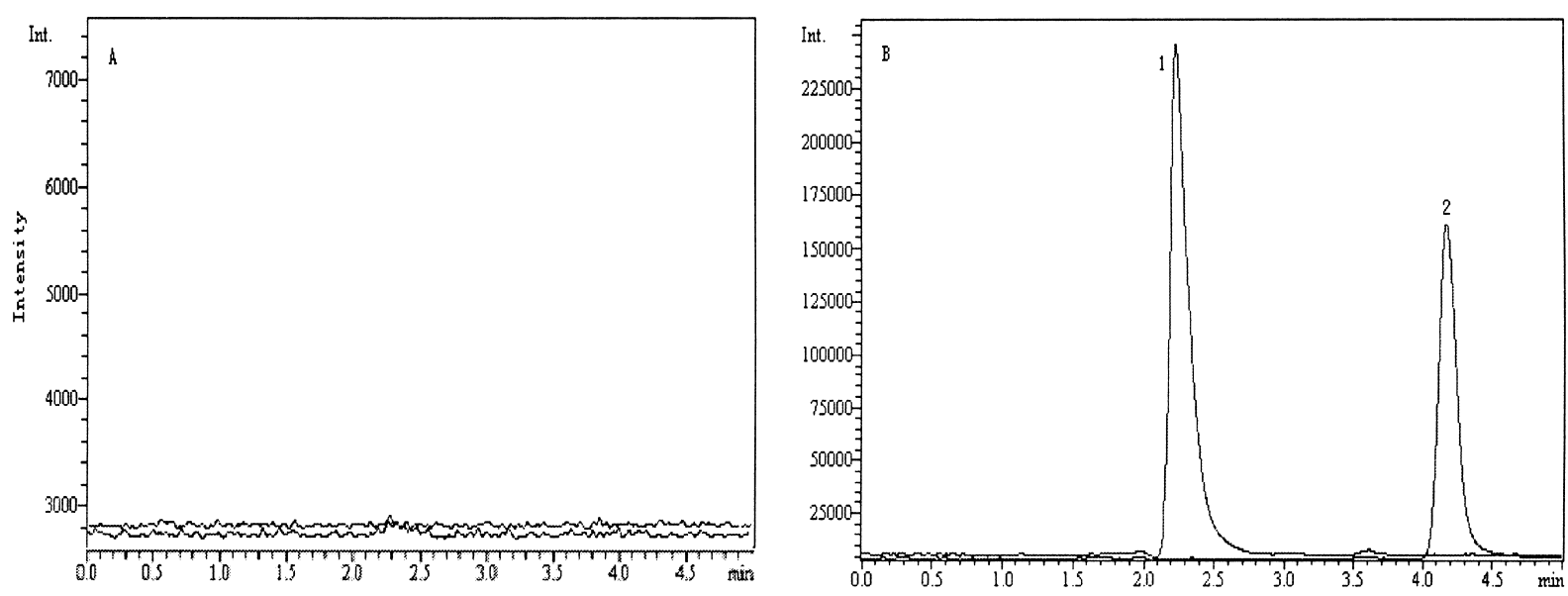

Fig. 2. Chromatograms of Guanfu Base I (GFI) Using LC-ESI-MS

(A) Blank plasma; (B) plasma sample after i.v. administration of GFI in rats. Peak 1, GFI; Peak 2, internal standard.

gression was then used to determine the linearity of the curves and calculate the slope, intercept, and correlation coefficient of the line for each of the analytes. The concentration of each sample was calculated based on the standard curve. All data is represented as mean \pm S.D.

\section{RESULTS}

Chromatography Using the described chromatographic method, symmetrical peaks were observed for GFI and internal standard. There were no peaks interfering with GFI or internal standard. The retention times of GFI and internal standard were 2.3 and $4.2 \mathrm{~min}$, respectively. The overall chromatographic run time was 5 min (Fig. 2).

Method Validation The linear range was calculated based on the peak area ratio of GFI to DDPH (I.S) versus the nominal concentration with the least-square regression. The calibration curve was linear from 0.05 to $20 \mu \mathrm{g} / \mathrm{ml} \quad(y=$ $0.1488 x+0.0835, r=0.9994)$; the absolute recovery of GFI was determined by direct comparison of peak area from extracts versus non-extracted samples. The mean recoveries for GFI were $86.8 \pm 6.7 \%, 93.5 \pm 5.4 \%$, and $90.2 \pm 2.3 \%$ at the $0.05,1.0$ and $20.0 \mu \mathrm{g} / \mathrm{ml}$, respectively $(n=5)$.

The intraday precision was determined by analyzing five samples spiked with GFI at concentrations of $0.05,1.0$ and $20.0 \mu \mathrm{g} / \mathrm{ml}$. The interday precision was determined by analyzing the spiked samples on 5 consecutive days. The intraday and interday precision was generally good $(<15 \%)$ throughout the assay range (Table 1).

Pharmacokinetic Parameters The plasma GFI concentration-time profiles following p.o. and i.v. administration of GFI are shown in Figs. 3 and 4, respectively. Pharmacokinetic parameters were estimated using a 3 p 87 software package. ${ }^{10)}$ Maximum concentration $\left(C_{\max }\right)$ and time to maximum concentration $\left(T_{\max }\right)$ were the experimentally observed values following oral administration of GFI. The area under the plasma concentration-time curve $(A U C)$ was calculated using the trapezoidal rule. $A U C_{t}$ was calculated from time zero to $12 \mathrm{~h}$ and $A U C_{\infty}$ from time zero to infinity. The terminal elimination half-life $\left(T_{1 / 2}\right)$ was obtained by dividing $\operatorname{Ln} 2$ by $\beta$. ( $\beta$ is the absolute value of the slope of the least-square regression line for the terminal datum point). The absolute
Table 1. Recovery and Precision of GFI in Plasma

\begin{tabular}{cccc}
\hline \hline \multirow{2}{*}{$\begin{array}{c}\text { Concentration } \\
\left(\mu \mathrm{g} \cdot \mathrm{ml}^{-1}\right)\end{array}$} & $\begin{array}{c}\text { Recovery } \\
(\%)\end{array}$ & \multicolumn{2}{c}{ Precision (RSD) $(\%)$} \\
\cline { 3 - 4 } & & Intraday & Interday \\
\hline 0.05 & $86.8 \pm 6.7$ & 9.1 & 13.6 \\
1.0 & $93.5 \pm 5.4$ & 7.3 & 11.8 \\
20.0 & $90.2 \pm 2.3$ & 7.4 & 7.6
\end{tabular}

Mean \pm S.D. $(n=5)$.

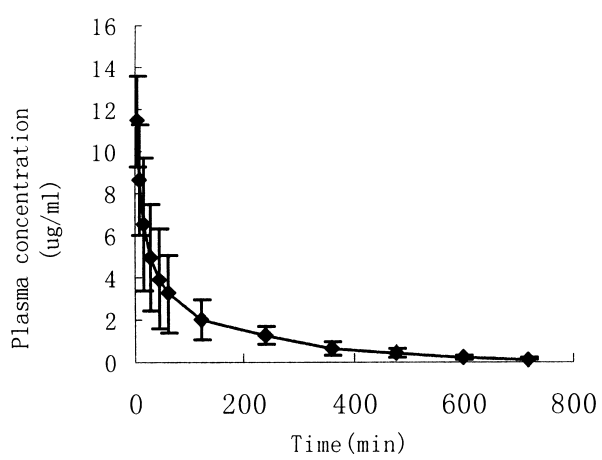

Fig. 3. Plasma Concentration-Time Profiles of GFI after i.v. Administration to Six Rats

Data represent mean \pm S.D.

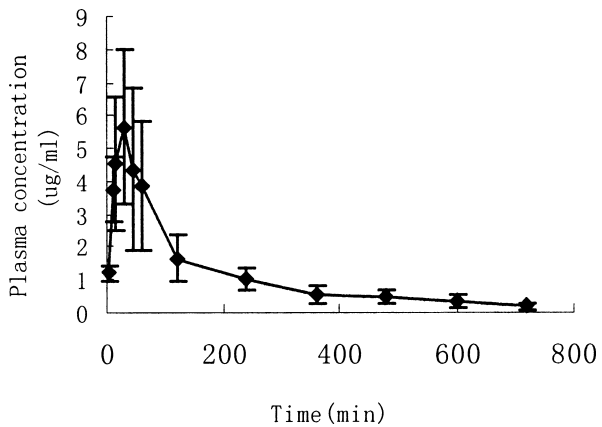

Fig. 4. Plasma Concentration-Time Profiles of GFI after Oral Administration to Six Rats

Data represent mean \pm S.D. 
Table 2. Mean Pharmacokinetic Parameters after Single Intravenous and Oral Doses of GFI $(20 \mathrm{mg} / \mathrm{kg})$ to Six Male Rats

\begin{tabular}{lcc}
\hline \hline Parameter & Intravenous & Oral \\
\hline$C_{\max }(\mu \mathrm{g} / \mathrm{ml})$ & - & $5.65 \pm 2.31$ \\
$T_{\max }(\mathrm{h})$ & - & $0.5 \pm 0.14$ \\
$A U C_{t}(\mathrm{mg} \cdot \mathrm{h} / \mathrm{l})$ & $15.66 \pm 4.92$ & $10.96 \pm 3.28$ \\
$A U C_{\infty}(\mathrm{mg} \cdot \mathrm{h} / \mathrm{l})$ & $15.70 \pm 5.41$ & $11.19 \pm 3.46$ \\
$T_{1 / 2}(\mathrm{~h})$ & $2.49 \pm 0.45$ & $2.74 \pm 0.87$ \\
$C L(1 / \mathrm{kg} / \mathrm{h})$ & $1.46 \pm 0.56$ & $1.38 \pm 0.47$ \\
$F \%$ & - & $71.31 \pm 16.82$ \\
\hline
\end{tabular}

bioavailability $(F \%)$ was estimated from the dose-normalized ratios of $\left[A U C_{\infty}\right]_{p . o}$ to $\left[A U C_{\infty}\right]_{\text {i.v. }}$ Plasma clearance $(C L)$ was calculated as $C L=\operatorname{Dose} / A U C_{\infty}$. The estimated pharmacokinetic parameters are shown in Table 2.

\section{DISCUSSION}

It has been reported that GFI is a metabolite of GFA in rats and humans, and preclinical pharmacological studies have showed that GFI has a potent anti-arrhythmic effect. Compared with GFI, GFA showed a much more potent anti-arrhythmic effect, while GFI had a less potent anti-arrhythmic effect with less toxicity. It is well known that drug metabolism plays an important role in pharmacodynamics and toxicity. In order to have a good understanding of GFA action, it is important to understand the pharmacokinetics of the parent compounds and their metabolites. The pharmacokinetics of GFA in dogs and humans have been reported. ${ }^{11,12)}$ In the present study, we determined the pharmacokinetics of GFI in rats. To the best of our knowledge, this is the first paper to describe the pharmacokinetics of GFI.

GFA and GFI are all esters of the same C20-diterpenoid structure. Their chemical structure is quite different from any of the anti-arrhythmic we are now using in the clinic. It is difficult to analyze GFI with high sensitivity using HPLC methods because of its weak UV absorption and lack of fluorescence in the molecular structure. In the study presented here, we established an analytical method using LC-ESI-MS. There was no interference in the blank plasma chromatograph. Baseline separation of GFI and the internal standard was achieved within $5 \mathrm{~min}$. Moreover, the sample extraction procedure was quite simple and stable.

This study describes the pharmacokinetic profiles of GFI in rats. The results indicated the drug plasma concentration curves, i.v. administration of GFI to rats, conformed to a twocompartment model of the first order absorption and a onecompartment model for p.o. administration. When GFI was administered orally to rats, the plasma level of GFI reached a maximum within $0.5 \mathrm{~h}$. After i.v. and p.o. administeration to rats, the terminal elimination half-life of GFI was $2.49 \mathrm{~h}$ and $2.74 \mathrm{~h}$, respectively. The absolute bioavailability of GFI was $71.31 \%$. These results indicate that GFI appeared to be rapidly absorbed and rapidly eliminated following both i.v. and p.o. administration. The bioavailability in rats was relatively higher.

The pharmacokinetic parameters of GFA in rats have not been reported. It has been reported that the main pharmacokinetics of GFA with i.v. administration in dogs and humans was similar, however, the terminal elimination half-life $\left(T_{1 / 2}\right)$ was different, with the $T_{1 / 2}$ in dogs ( $14 \mathrm{~h}$ ) being longer than in humans $(8 \mathrm{~h}) .^{11,12)}$ Our result for the $T_{1 / 2}$ of GFI in rats was $2.5 \mathrm{~h}$, which is much shorter than the $T_{1 / 2}$ of GFA in dogs and humans. One reason of this probably is due to different race. With a longer $T_{1 / 2}$, administration of GFA in clinics probably has a longer internal.

GFA and GFI have the same molecular structure and only differ in the number of acetyls. GFA has two acetyls and GFI has one acetyl. It is well known that the hydrolysis of esters can take place in plasma by a non-specific esterase or in liver by aspecific esterase. It has been reported that GFI was found in bile after intravenous GFA administration in rats. ${ }^{7)}$ However, it was not clear whether GFI, which was found in rat bile, was a hydrolyte or metabolite of GFA. In our experiments, after i.v. or p.o. administration of GFI, GFA was not found in plasma in the scan and SIM modes, suggesting that GFI may be a hydrolyte or a metabolite of GFA. However GFI most likely cannot be changed to GFA in rats. We are currently conducting further experiments to study the mechanism of metabolism of GFA.

\section{CONCLUSION}

A specific, sensitive, rapid, precise and accurate LC-ESIMS assay for the determination of GFI was developed and used to estimate its pharmacokinetic parameters. The results of the present study show that the absorption and elimination of GFI was relatively rapid and the bioavailability in rats was relatively high. The results of the present study can hopefully be applicable to clinical pharmacokinetic studies.

Acknowledgments The authors wish to thank Prof. Longsheng Sheng (Center of Drug Analysis, China Pharmaceutical University, Nanjing, China) for his technical assistance during the determination of GFI by LC-MS. This study was supported by National "863" Project (No.2003AA2Z347A, No.2002AA2Z3102)

\section{REFERENCES}

1) Liu J. H., Wang H. C., Cao Y. L., Chin. Tradit. Herb Drugs, 12, 1-2 (1981).

2) Hou D. H., Li L., Wang Q. J., J. Nanjing Coll. Pharm., 2, 68-74 (1981).

3) Chen W. J., Dong Y. L., Zhang Y. F., Ding G. S., Acta Pharmacol. Sinica, 4, 247-250 (1983).

4) Wang Y. P., Chen W. Z., Gu P. K., Acta Pharmacol. Sinica, 11, 502505 (1990).

5) Chen H. Z., Chen W. Z., Gu P. K., Zhang L., Jin Z. J., Acta Pharmacol. Sinica, 10, 399-401 (1989).

6) Zhang L., Gu P. K., Zao W. B., Chen Y. L., Zhang J. X., Jin Z. J., Acta Pharmacol. Sinica, 7, 234-236 (1986).

7) A J. Y., Wang G. J., Liu X. Q., Jiang D. Y., Liu J. H., Acta Pharmaceutica Sinica, 37, 283-287 (2002).

8) A J. Y., Wang G. J., Sun J. G., Gu Y. C., Wu M. S., Liu J. H., Eur. J. Drug Metab. Pharmacokinet., 28, 265-272 (2003).

9) Hou D. H., Wu L. Y., Xu W. R., Liu J. H., China Pharm. Univ., 18, 268-272 (1987).

10) Yang Y., Zhang W., Tang Z., J. Nanjing Univ. (Nat. Sci. Ed. II), 30, 63-65 (1994).

11) Wu M. S., Wang G. J., Cai X. H., Sun J. G., Liu J. H., Acta Pharmaceutica Sinica, 37, 551-554 (2002).

12) Wang G. G., Cui H. D., Zhu J., Li P., Yang Y. M., Chin. J. Clin. Pharmacol., 16, 283-258 (2000). 